\title{
Fragment-Modified Graphene FET for Highly Sensitive Detection of Antigen-Antibody Reaction
}

\author{
Shogo Okamoto, Yasuhide Ohno, Kenzo Maehashi, Koichi Inoue and Kazuhiko Matsumoto \\ The Institute of Scientific and Industrial Research, Osaka University \\ 8-1 Mihogaoka, Ibaraki, Osaka 567-0047, Japan \\ E-mail:okamot11@sanken.osaka-u.ac.jp
}

\begin{abstract}
For high-sensitive and specific protein sensing using graphene field-effect transistors (G-FETs), the antigen-binding fragment (Fab), which is a component of conventional antibody, was functionalized onto the graphene surface. Since the height of the Fab is approximately $3 \mathrm{~nm}$, the antigen-antibody reaction is expected to occur inside the electrical-double layer in the buffer solution. After functionalization of Fab onto the G-FET, the transfer characteristics shifted in the positive gate-voltage direction, indicating that the Fab was successfully modified onto the graphene surface. And then, plots of the conductance change and the target proteins concentration were fitted by the Langmuir adsorption isotherm. These results indicate that the Fab-modified G-FETs have high potentials for high sensitive biological sensors using antigen-antibody reactions.
\end{abstract}

Key words:specific protein sensing, graphene field-effect transistors,antigen-binding fragment,electrical-double layer,biological sensors

\section{Introduction}

Graphene is a two-dimensional material and its unique electrical characteristics show high potential for sensing applications [1-6]. Labelfree biosensors based on graphene field-effect transistors (G-FETs) with high sensitivity have been reported in recent years [7-11]. In the general FET-based biosensing measurements, the formation of the electrical-double layer is very important concept [12-14]. The mobile charges in the transistor's channel no longer feel the charged molecules placed more than the Debye length away. Since the Debye length is basically very small $(\sim 5 \mathrm{~nm})$, it is difficult for FETs to detect the antigen using whole antibody $(\sim 15 \mathrm{~nm})$. Therefore, we used antigenbinding fragment (Fab: $\sim 3 \mathrm{~nm}$ ) as shown in Fig. 1 [15]. In this study, Fab-modified G-FETs were fabricated to detect the specific proteins with high sensitivity.

\section{Experimental Procedure}

G-FETs were fabricated on a thermally oxidized 280-nm-thick $\mathrm{SiO}_{2}$ layer on the degenerately doped $\mathrm{Si}$ substrates. Monolayer graphene flakes were only used for the channel in this work to obtain a high sensitivity, and they were prepared using bulk graphite and a clear adhesive tape. After the layer number was confirmed by Raman spectroscopy $[16,17]$, the source and drain gold electrodes were formed by conventional electron-beam lithography, vacuum evaporation, and the lift-off method. An optical image of a G-FET is shown in Fig. 2.In order to perform sensing measurements, The silicone rubber container was put on the graphene channel to prevent solution leakage. And an $\mathrm{Ag} / \mathrm{AgCl}$ reference electrode was used for applying top-gate voltage. Figure 3 shows an experimental setup of the G-FET in a solution.

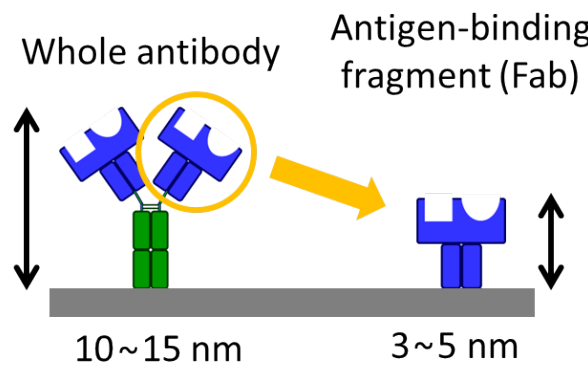

Fig. 1. Schematic of a whole antibody and an antigen-binding fragment.

The following is a fabrication process of Fabmodified G-FETs. First, the G-FETs were immersed in 1-pyrenebutanoic acid succinimidyl ester, which was used as linkers [18-20]. Then, for the covalent immobilization of the Fab on 
the graphene surface, the devices were immersed in Fab in phosphate-buffered saline (PBS). In this work, heat shock proteins (HSP) were used as a target protein $[21,22]$.

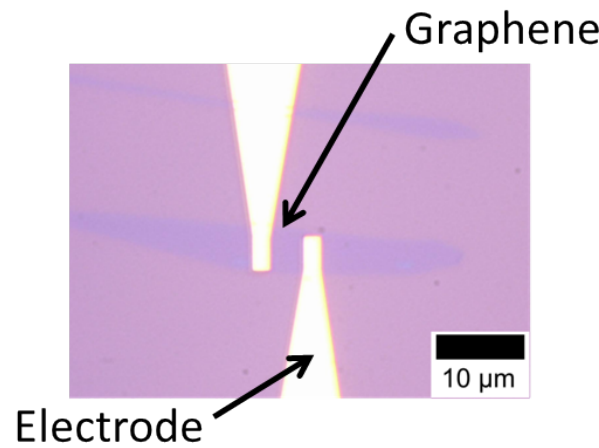

Fig. 2. Optical microscopy image of a G-FET.

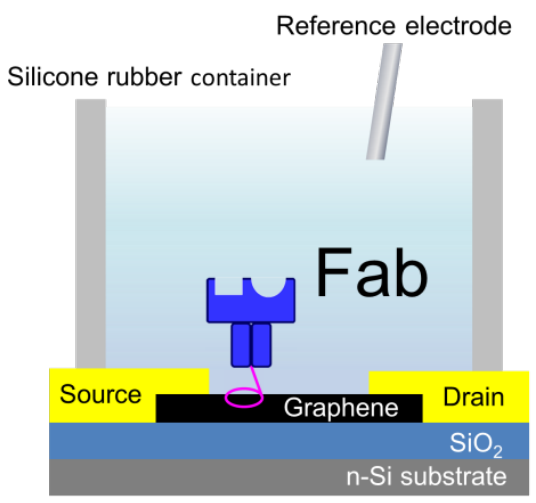

Fig. 3. Schematic image of a measurement system.

\section{Results and Discussion}

The conductance $(C)$ versus top-gate voltage $\left(V_{\mathrm{TG}}\right)$ characteristics for the G-FET with bare graphene channel and Fab-modified graphene channel in PBS are shown in Fig. 4. The $C-V_{\text {TG }}$ curves showed an ambipolar characteristic, indicating that the Fermi level in graphene was changed by the $V_{\text {TG }}$. After functionalization of Fab, transfer characteristics were shifted in the positive direction, indicating that the Fab was successfully functionalized on the graphene channel. This positive shift can be considered owing to the Fermi level shift. Thus, it is considered that Fab has negative charge in PBS at $\mathrm{pH}$ 7.4. Furthermore, the $C-V_{\mathrm{TG}}$ characteristics have little change in slope, indicating that the Fab-fabrication process was carried out without introducing defects on the graphene surface.

To confirm the antigen-antibody reaction, the target HSP concentration dependence was measured and the dissociation constant $\left(K_{\mathrm{D}}\right)$ between Fabs and HSPs was estimated. Figure 5 shows the time dependence of $C$ at the drain voltage $\left(V_{\mathrm{D}}\right)=0.1 \mathrm{~V}$ and $V_{\mathrm{TG}}=-0.05 \mathrm{~V}$. Every ten min, the HSPs at various concentrations were introduced onto the Fab-modified G-FETs. The concentrations of HSPs were $0.01,0.1,0.8,7$, 10,60 , and $100 \mathrm{nM}$. The stepwise decrease in $C$ was clearly observed after the injections of the HSPs at various concentrations. The $C$ for each HSP concentration was maintained at an approximately constant value. In this device, since the carriers were holes at $V_{\mathrm{TG}}=-0.05 \mathrm{~V}$, the decreased $C$ indicates that the Fabmodified G-FETs detected the positive charges of the HSPs in the electrical double layer. This concentration dependence reveals that the GFET detects HSPs at concentrations as low as $100 \mathrm{pM}$, and that dynamic range of HSP detection is several hundreds of $\mathrm{pM}$ to hundreds of $\mathrm{nM}$, indicating the high sensitivity of Fab-modified G-FETs [23].

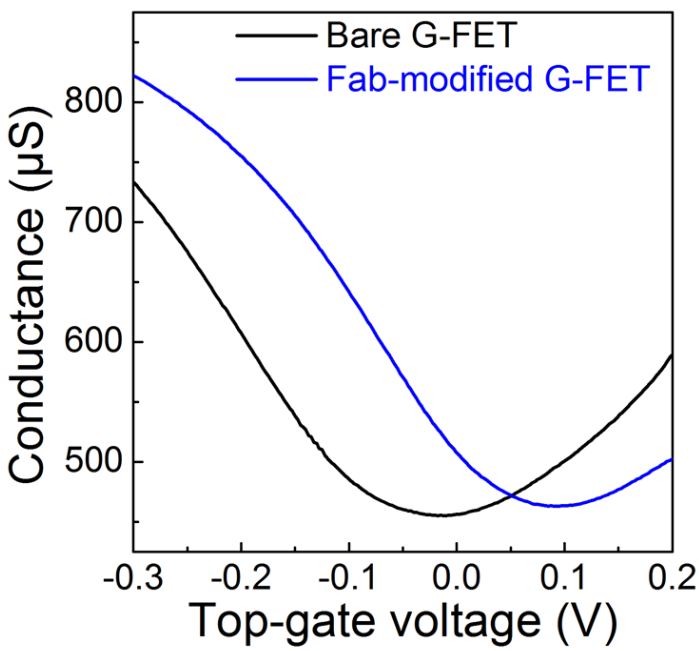

Fig. 4. Transfer characteristics of the G-FET for as fabricated (black line) and after modification of Fab (blue line).

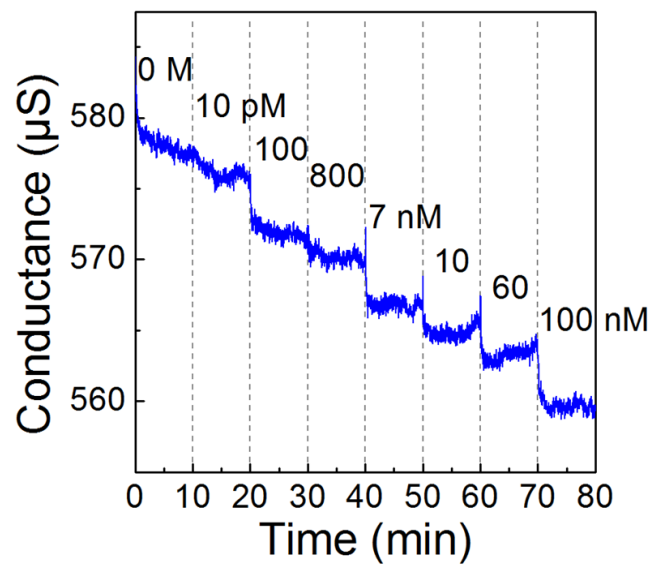

Fig. 5. Time course of $I_{D}$ for the Fab-modified GFET at $V_{D}$ of $0.1 \mathrm{~V}$ and $V_{T G}$ of $-0.05 \mathrm{~V}$. At $10 \mathrm{~min}$ intervals, various concentrations of HSPS were introduced on the Fab-modified G-FET. 
Then, Figure 6 shows the net change in the conductance $(\Delta C)$ plotted as a function of HSP concentration $\left(C_{\mathrm{HSP}}\right)$. The data indicates that the reaction between $\mathrm{Fab}$ and HSP in the graphene channel follows the Langmuir adsorption isotherm $[8,24]$. The curve was fitted by the data points. From Fig. $6, K_{D}$ was estimated to be $2.3 \mathrm{nM}$. These sensing characteristics show that the Fab-modified GFETs detects the antigen-antibody reaction on the surface, and highly sensitive biological sensors can be realized with G-FETs using Fab.

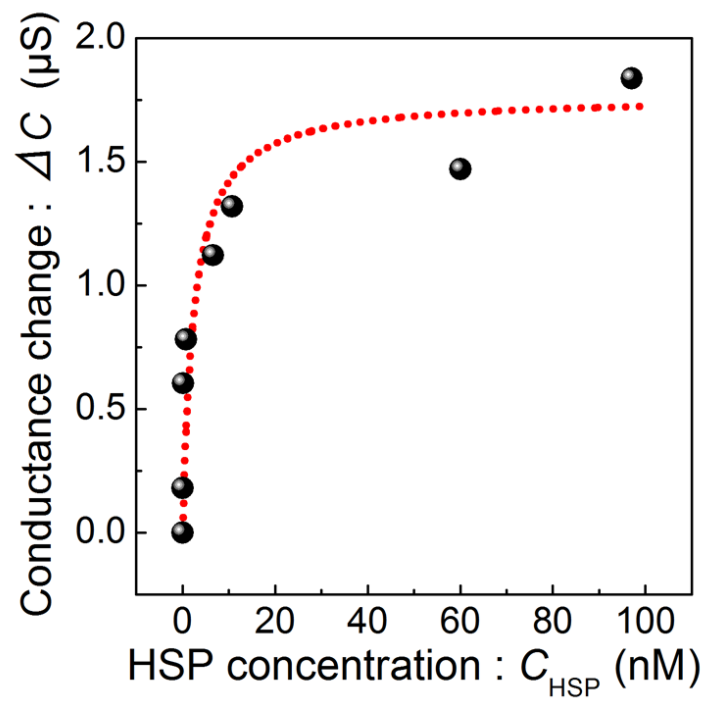

Fig. 6. Conductance change versus HSP concentration. The red dashed line shows a fitting curve to the Langmuir adsorption isotherm.

\section{Conclusions}

We have fabricated Fab-modified G-FETs for biological sensors to utilize the antigen-antibody reaction on the graphene channel surface. The $I_{D}-V_{\text {TG }}$ curve shifted in the positive direction without changes in the transconductances of these G-FETs following the functionalization of Fabs, indicating that Fabs were successfully immobilized without the introduction of defects on the graphene surface. The dissociation constant was estimated to be $2.3 \mathrm{nM}$ on the basis of the HSP concentration dependence. The results show that we detect HSPs with high sensitivity without the need for the labeling process. The use of Fabs has several advantages in biosensors based on G-FETs. Fabs are found in all of the antibodies; therefore, it is expected that Fab-modified G-FETs can improve disease diagnosis. Therefore, Fabmodified G-FETs are promising devices for highly sensitive biological sensors.

\section{Acknowledgements}

The authors acknowledge the generous support from the New Energy and Industrial Technology
Development Organization (NEDO), the Core Research for Evolutional Science and Technology (CREST) from the Japan Science and Technology Agency (JST), Management Expenses Grants for National University Corporations from MEXT, and Japan Society for the Promotion of Science Core-to-Core Program. The authors thank Messrs. W. Huang and $\mathrm{T}$. Tang of Abnova Corporation (Taiwan) for providing us the Fabs and target proteins.

\section{References}

[1] K. S. Novoselov, A. K. Geim, S. V. Morozov, D. Jiang, Y. Zhang, S. V. Dubonos, I. V. Grigorieva, and A. A. Firsov, Electric Field Effect in Atomically Thin Carbon Films, Science 306, 666669 (2004); doi: 10.1126/science.1102896

[2] A. K. Geim and K. S. Novoselov, The rise of graphene,Nature Materials 6 183-191 (2007); doi: 10.1038/nmat1849

[3] K. I. Bolotina, K. J. Sikesb, Z. Jianga, M. Klimac, G. Fudenberga, J. Honec, P. Kima, and H. L. Stormer, Ultrahigh electron mobility in suspended graphene, Solid State Communications 146, 351355 (2008); doi: 10.1016/j.ssc.2008.02.024

[4] T. O. Wehling, K. S. Novoselov, S. V. Morozov, E. E. Vdovin, M. I. Katsnelson, A. K. Geim, and A. I. Lichtenstein, Molecular Doping of Graphene, $\begin{array}{llll}\text { Nano Letters } 8 & 173-177 & \text { (2008); doi: }\end{array}$ $10.1021 / \mathrm{nl072364w}$

[5] K. Nagashio, T. Nishimura, K. Kita, and A. Toriumi, Systematic Investigation of the Intrinsic Channel Properties and Contact Resistance of Monolayer and Multilayer Graphene Field-Effect Transistor, Japanese Journal of Applied Physics 49051304-1-6 (2010);

doi: 10.1143/JJAP.49.051304

[6] K. Nagashio, T. Nishimura, K. Kita, and A. Toriumi, Contact resistivity and current flow path at metal/graphene contact,Applied Physics Letters 97, 143514-1-3 (2010); doi: 10.1063/1.3491804

[7] Y. Ohno, K. Maehashi, Y. Yamashiro, and K. Matsumoto, Electrolyte-Gated Graphene FieldEffect Transistors for Detecting $\mathrm{pH}$ and Protein Adsorption,Nano Letters 9, 3318-3322 (2009); doi: 10.1021/nl901596m

[8] Y. Ohno, K. Maehashi, and K. Matsumoto, LabelFree Biosensors Based on Aptamer-Modified Graphene Field-Effect Transistors, Journal of the American Chemical Society 132, 18012-18013 (2010); doi: 10.1021/ja108127r

[9] Y. Sofue, Y. Ohno, K. Maehashi, K. Inoue, and K. Matsumoto, Highly Sensitive Electrical Detection of Sodium lons Based on Graphene Field-Effect Transistors, Japanese Journal of Applied Physics 50, 06GE07-1-4 (2011); doi: 10.1143/JJAP.50.06GE07

[10] S. Mao. G. Lu, K. Yu, Z. Bo, and J. Chen,Specific Protein Detection Using Thermally Reduced 
Graphene Oxide Sheet Decorated with Gold Nanoparticle-Antibody Conjugates,Advanced Materials 22, 3521-3526 (2010); doi: 10.1002/adma.201000520

[11] S. Mao, K. Yu, G. Lu, and J. Chen,Highly Sensitive Protein Sensor Based on ThermallyReduced Graphene Oxide Field-Effect Transistor,Nano Research 4, 921-930 (2011);doi: $10.1007 / \mathrm{s} 12274-011-0148-3$

[12] M. Krüger, M. R. Buitelaar, T. Nussbaumer, C. Schönenberger, and L. Forró,Electrochemical carbon nanotube field-effect transistor,Applied Physics Letters 78,1291-1293 (2001); doi: 10.1063/1.1350427

[13] S. Rosenblatt, Y. Yaish, J. Park, J. Gore, V. Sazonova, and P. L. McEuen, High Performance Electrolyte Gated Carbon Nanotube Transistors, Nano Letters 2, 869-872 (2002);doi: 10.1021/nI025639a

[14] K. Maehashi, T.Katsura, K. Kerman, Y.Takamura, K. Matsumoto, andE. Tamiya, Label-Free Protein Biosensor Based on Aptamer-Modified Carbon Nanotube Field-Effect Transistors, Analytical Chemistry79, 782-787 (2007); doi: $10.1021 / \mathrm{ac} 060830 \mathrm{~g}$

[15] J. P. Kim, B. Y. Lee, S. Hong, S. J. Sim,Ultrasensitive carbon nanotube-based biosensors using antibody-binding fragments,Analytical Biochemistry 381,193-198 (2008); doi: 10.1016/j.ab.2008.06.040

[16] A. Ferrari, J. Meyer, V. Scardaci, C. Casiraghi, M. Lazzeri, F. Mauri, S. Piscanec, D. Jiang, K. Novoselov, S. Roth, and A. Geim,Raman Spectrum of Graphene and Graphene Layers, Physical Review Letters 97, 187401-1-4 (2006); doi: 10.1103/PhysRevLett.97.187401

[17] Y. Ohno, K. Maehashi, and K. Matsumoto, Chemical and biological sensing applications based on graphene field-effect transistors,
Biosensors and Bioelectronics 26, 1727-1730 (2010); doi: 10.1016/j.bios.2010.08.001

[18] R. J. Chen, Y. Zhang, D. Wang, and $H$. Dai,Noncovalent Sidewall Functionalization of Single-Walled Carbon Nanotubes for Protein Immobilization, Journal ofAmerican Chemical Society 123,3838-3839 (2001); doi: 10.1021/ja010172b

[19] T. Li, M. Yang, and H. Li, Label-free electrochemical detection of cancer marker based on graphene-cobalt hexacyanoferratenanocomposite,Journal of Electroanalytical Chemistry 655, 50-55 (2011); doi: 10.1016/j.jelechem.2011.02.009

[20] S. R. Guo, J. Lin, M. Penchev, E. Yengel, M. Ghazinejad, C. S. Ozkan, and M. Ozkan,Label Free DNA Detection Using Large Area Graphene Based Field Effect Transistor Biosensors,Journal of Nanoscience and Nanotechnology 11,52585263 (2011); doi: 10.1166/jnn.2011.3885

[21] S. K. Calderwood, M. A. Khaleque, D. B. Sawyer, and D. R. Ciocca, Heat shock proteins in cancer: chaperones of tumorigenesis, TRENDS in Biochemical Sciences 31, 164-172 (2006); doi: 10.1016/j.tibs.2006.01.006

[22] J. S. Hahn, The Hsp90 chaperone machinery: from structure to drug development,BMB Reports 42 623-630 (2009)

[23] O. S. Kwona, T. J. Honga, S. K. Kima, J. H. Jeongb, J. S Hahna, and J. Janga, Hsp90functionalized polypyrrole nanotube FET sensor for anti-cancer agent detection,Biosensors and Bioelectronics 25, 1307-1312 (2010); doi: 10.1016/j.bios.2009.10.019

[24] K. Maehashi, K. Matsumoto, Y. Takamura, and E. Tamiya, Aptamer-Based Label-Free Immunosensors Using Carbon Nanotube FieldEffect Transistors,Electroanalysis 21, 1285-1290 (2009); doi: 10.1002/elan.200804552 